\title{
Novel Method to Improve the Signal-to-Noise Ratio in Far-Field Results Obtained from Planar Near-Field Measurements
}

\author{
Francisco J. Cano-Fácila', Sara Burgos ${ }^{2}$, and Manuel Sierra-Castañer ${ }^{1}$ \\ ${ }^{1}$ Universidad Politécnica de Madrid (UPM), ETSI de Telecomunicación \\ Av/ Complutense 30, 28040 Madrid, Spain \\ E-mail: francisco@gr.ssr.upm.es, mscastaner@gr.ssr.upm.es \\ ${ }^{2}$ Antenna Systems Solutions, S.L. (ASYSOL) \\ c/ Manuel Cortina 12, 28010, Madrid, Spain \\ E-mail: sburgos@asysol.com
}

\begin{abstract}
A method to reduce the noise power in a far-field pattern without modifying the desired signal is proposed. An important signal-to-noise-ratio improvement may thereby be achieved. The method is used when the antenna measurement is performed in the planar near-field, where the recorded data are assumed to be corrupted with white Gaussian and spacestationary noise, because of the receiver's additive noise. Back-propagating the measured field from the scan plane to the plane of the antenna under test (AUT), the noise remains white Gaussian and space-stationary, whereas the desired field is theoretically concentrated in the aperture antenna. Thanks to this fact, a spatial filtering may be applied, canceling the field that is located out of the AUT's dimensions and that is only composed of noise. Next, a planar-field-to-far-field transformation is carried out, achieving a great improvement compared to the pattern obtained directly from the measurement. To verify the effectiveness of the method, two examples are presented using both simulated and measured near-field data.
\end{abstract}

Keywords: Antenna measurements; signal to noise ratio; Gaussian noise; filtering; reconstruction algorithms; anechoic chambers; random variables

\section{Introduction}

$\mathbf{N}$ ear-field measurement has become one of the most commonly employed techniques for obtaining antenna radiation patterns. In contrast to conventional far-field ranges, the distance between the antenna under test (AUT) and the probe is reduced. Unwanted contributions from reflections or diffraction from the environment are largely suppressed in the anechoic chambers in which these measurements are typically performed. Moreover, accurate farfield results can be obtained from near-field data by using either a modal-expansion method $[1,2]$ or an equivalent-current reconstruction method $[3,4]$. These techniques yield far-field results that are in many cases more accurate than those obtained in a far-field range. Nevertheless, as in all measurements, there are always sources of error that must be taken into account in near-field antenna measurements. In light of such potential errors, the effect of measurement error on the final result cannot be immediately deduced. The quantity to be determined differs from the measured quantity, and an uncertainty analysis is required to assess the effect of near-field errors on the accuracy of the far-field pattern. References [5-10] are examples of studies that examined the relation- ships between the measurement errors and their effects on the far field using mathematical analyses, simulations, or measurements. The results obtained in these studies can be used to estimate the impact of a particular error or a combination of errors on the farfield pattern. In addition, the results can be used to deduce the maximum admissible near-field error for a given level of accuracy in the far field, or to assess the accuracy of a near-field range [11].

Random noise is one of the errors that limit the accuracy of far-field results, particularly when measuring a low-sidelobe or a high-performance antenna. One possible source for this error is receiver noise, which is present in all measurements. Some comprehensive studies for random noise in near-field measurements have already been presented. However, their results are valid for only a particular measurement system (planar, cylindrical, or spherical), due to the different near-field-to-far-field transformation processes required for each of them. For the planar system, two independent analyses with similar results were proposed in $[12,13]$. Both of these started with random errors in the planar near field, and obtained expressions that represented the signal-tonoise ratio in the far field as a function of the noise power in the near field. A similar study for cylindrical near-field measurements 
was carried out in $[14,15]$. These latter publications derived an expression relating the noise power in the near field and far field. Furthermore, they analyzed the effect of the noise in the far field, and present expressions for the boundaries that defined a strip where it was possible to find the radiation pattern for a given probability. The cylindrical case was also discussed in [16], which investigated the improvement of the signal-to-noise ratio achieved through the cylindrical near-field-to-far-field transformation.

This paper focuses on the planar near-field case. Here, the measurement is assumed to be corrupted by complex white Gaussian and space-stationary noise, as in $[14,15]$. In contrast to previous studies, we perform a statistical analysis of the random noise, and propose an algorithm to reduce the noise power in the far field to improve the signal-to-noise ratio. Increasing the signalto-noise ratio by reducing the noise power was proposed in [17, 18] for the spherical near-field case. Those studies also presented a technique for canceling a greater portion of the noise by over-sampling to obtain a higher number of spherical modes than required [19], and thus to be able to apply a modal filtering. In this paper, noise reduction is achieved with noise filtering; however, here spatial filtering is employed instead of a modal filtering.

This paper is organized as follows. Section 2 gives an overview of the back-propagation process to obtain the field at the AUT's plane from the measured field. The effect of the backpropagation process on the random noise added to the measured data is also analyzed in this section. Section 3 describes the method implemented to improve the signal-to-noise ratio. Section 4 presents two numerical results for analyzing the effectiveness of the method. Finally, conclusions are discussed in Section 5.

\section{Back-Propagation of the Planar Near Field}

As previously stated, the objective of this paper is to mitigate the undesired effects of random noise when the measurement is performed in the planar near field. This noise mitigation is accomplished by means of filtering before obtaining the far-field results. Because all the measured data are always noise corrupted, filtering cannot be applied to this initial information, and a new data representation that allows for noise filtering without canceling out the desired information is needed. For this, once the planar near-field measurement has been performed, the field at the AUT's plane (the reconstructed field) is computed. Because the desired contribution is theoretically located inside the dimensions of the AUT, filtering can be applied to cancel the outside contribution due to noise.

\subsection{Theoretical Description of the Transformation}

In a planar near-field measurement, the scan plane and the AUT plane are always parallel. Therefore, an easy transformation from one plane to the other can be performed using field backpropagation $[20,21]$. Assuming that the normal axis to both planes is the $z$ axis and that the distance between them is $d$, the measured near-field components are $E_{\text {meas }, x}(x, y, d)$ and $E_{\text {meas }, y}(x, y, d)$. In addition, the plane-wave spectrum (PWS) components referenced to the scan plane, $P_{x}\left(k_{x}, k_{y}, d\right)$ and $P_{y}\left(k_{x}, k_{y}, d\right)$, are calculated as follows:

$$
\begin{aligned}
& P_{x}\left(k_{x}, k_{y}, d\right)=\frac{1}{2 \pi} \iint E_{\text {meas }, x}(x, y, d) e^{j\left(k_{x} x+k_{y} y\right)} d x d y, \\
& P_{y}\left(k_{x}, k_{y}, d\right)=\frac{1}{2 \pi} \iint E_{\text {meas }, y}(x, y, d) e^{j\left(k_{x} x+k_{y} y\right)} d x d y .
\end{aligned}
$$

The next step in calculating the reconstructed field is to reference the last quantities given by Equation (1) to the AUT's plane. Each plane wave is multiplied by a term that depends on the distance between planes as well as the longitudinal component of the propagation vector, $k_{z}=\sqrt{k_{0}^{2}-k_{x}^{2}-k_{y}^{2}}$ :

$$
\begin{aligned}
& P_{x}\left(k_{x}, k_{y}, 0\right)=P_{x}\left(k_{x}, k_{y}, d\right) e^{j k_{z} d}, \\
& P_{y}\left(k_{x}, k_{y}, 0\right)=P_{y}\left(k_{x}, k_{y}, d\right) e^{j k_{z} d} .
\end{aligned}
$$

Finally, using the inverse expression of Equation (1), the electricfield components over the AUT's plane, $E_{a p, x}(x, y, 0)$ and $E_{a p, y}(x, y, 0)$, can be computed:

$$
\begin{aligned}
& E_{a p, x}(x, y, 0)=\frac{1}{2 \pi} \iint P_{x}\left(k_{x}, k_{y}, 0\right) e^{-j\left(k_{x} x+k_{y} y\right)} d k_{x} d k_{y} \\
& E_{a p, y}(x, y, 0)=\frac{1}{2 \pi} \iint P_{y}\left(k_{x}, k_{y}, 0\right) e^{-j\left(k_{x} x+k_{y} y\right)} d k_{x} d k_{y}
\end{aligned}
$$

\subsection{Noise Analysis in the Back-Propagation Process}

After reviewing the theory behind the planar near-field-toreconstructed-field transformation, an analysis to assess the noise behavior and to obtain its statistical parameters was carried out. In the analysis, complex white Gaussian and space-stationary noise was considered. Its mean and variance were assumed to be zero and $\sigma_{n f}^{2}$, respectively. Although not all noise sources are Gaussian, the Gaussian noise model is ideal for modeling the main noise contributions that limit the dynamic range. Moreover, because all the expressions are linear for the back-propagation process, the analysis was performed by considering only the noise. In addition, the expressions and the noise are the same for both electric-field polarizations. As a result, the study is developed for a generic case.

Using planar near-field data containing only noise and applying the discrete version of Equation (1), the plane-wave spectrum referenced to the scan plane due to the noise can be obtained:

$$
N\left(k_{x}, k_{y}, d\right)=\frac{\Delta x \Delta y}{2 \pi} \sum_{i=1}^{M} n_{n f}\left(x_{i}, y_{i}, d\right) e^{j\left(k_{x} x_{i}+k_{y} y_{i}\right)} \text {, }
$$

where $n_{n f}\left(x_{i}, y_{i}, d\right)$ represents the noise added to every near-field data point, $N\left(k_{x}, k_{y}, d\right)$ is the plane-wave spectrum referenced to the scan plane, $\Delta_{x}$ and $\Delta_{y}$ symbolize the sample spacing in the $x$ and $y$ directions, and $M$ is the total number of planar near-field samples. Because noise is an independent random variable at each measurement point, the plane-wave spectrum obtained from Equation (4) is also modeled as Gaussian and space-stationarity noise 
with zero mean. The variance is determined by calculating the autocorrelation in $(0,0), R_{N}(0,0)$ :

$$
\begin{aligned}
\sigma_{N}^{2} & =R_{N}(0,0)=E\left[N\left(k_{x}, k_{y}, d\right) N^{*}\left(k_{x}, k_{y}, d\right)\right] \\
& =M\left(\frac{\Delta x \Delta y}{2 \pi}\right)^{2} \sigma_{n f}^{2} .
\end{aligned}
$$

The next step in the transformation process is to reference the previous plane-wave spectrum to the AUT's plane. To do this, the plane-wave spectrum is multiplied by a complex factor of unity amplitude, as shown in Equation (6). The resulting quantity is also another Gaussian noise with the same statistical properties:

$$
N\left(k_{x}, k_{y}, 0\right)=N\left(k_{x}, k_{y}, d\right) e^{j k_{z} d}
$$

Finally, the reconstructed field is obtained by using the discrete version of Equation (3):

$$
n_{a p}(x, y, 0)=\frac{\Delta k_{x} \Delta k_{y}}{2 \pi} \sum_{m=1}^{M_{k}} N\left(k_{x, m}, k_{y, m}, 0\right) e^{-j\left(k_{x, m} x+k_{y, m} y\right)}
$$

$$
\begin{aligned}
= & \frac{\Delta k_{x} \Delta k_{y}}{2 \pi} \frac{\Delta x \Delta y}{2 \pi} \\
& \sum_{m=1}^{M_{k}} e^{j k_{x, m} d} \sum_{i=1}^{M} n_{n f}\left(x_{i}, y_{i}, d\right) e^{j\left(k_{x, m} x_{i}+k_{y, m} y_{i}\right)} e^{-j\left(k_{x, m} x+k_{y, m} y\right)}
\end{aligned}
$$

where $n_{a p}(x, y, 0)$ is the field over the AUT's plane due to noise, $\Delta k_{x}$ and $\Delta k_{y}$ represent the spectral steps in the $k_{x}$ and $k_{y}$ directions, and $M_{k}$ stands for the total number of spectral samples. The total number of spectral samples is equal to $M$ because the last summations in Equations (4) and (7) are evaluated using the fast Fourier transform (FFT) algorithm.

From Equation (7), it is deduced that the field at each point of the reconstructed plane is also a Gaussian random variable with zero mean and variance calculated as in Equation (5), i.e., by determining the autocorrelation in $(0,0)$ :

$$
\begin{aligned}
\sigma_{n_{a p}}^{2} & =R_{n_{a p}}(0,0)=E\left[n_{a p}(x, y, 0) n_{a p}^{*}(x, y, 0)\right] \\
& =\left(\frac{\Delta x M_{x}}{2 \pi}\right)^{2} \Delta k_{x}^{2}\left(\frac{\Delta y M_{y}}{2 \pi}\right)^{2} \Delta k_{y}^{2} \sigma_{n f}^{2}=\sigma_{n f}^{2},
\end{aligned}
$$

where the following relationships have been taken into account:

$$
\begin{aligned}
& M_{k}=M, \\
& M=M_{x} M_{y}, \\
& \Delta k_{x}=\frac{2 \pi}{\Delta x M_{x}}, \\
& \Delta k_{y}=\frac{2 \pi}{\Delta y M_{y}},
\end{aligned}
$$

where $M_{x}$ and $M_{y}$ represent the number of planar near-field samples in the $x$ and $y$ directions.

From the previous analysis, it can be seen that for planar near-field noise with the aforementioned statistical characteristics, the noise in both the plane-wave spectrum and in the reconstructed field is complex, stationary, white Gaussian noise, with zero mean and variance given by Equations (5) and (8), respectively, as shown Figure 1.

\section{Description of the Method}

As mentioned before, the main purpose of the proposed method is to reduce the far-field noise power obtained in a planar near-field measurement. However, noise reduction cannot be achieved at the input, because both the noise and the desired contribution are distributed over the whole measurement surface. For this reason, a field transformation is required to filter out a portion of the noise without modifying the desired signal. This paper presents a method that uses back-propagation of the field from the scan plane to the AUT's surface, and then a spatial filtering is applied. The specific steps are described in the following.

- The planar near-field data are used to calculate the plane-wave spectrum referenced to the scan plane by means of an inverse Fourier transform.

- The plane-wave spectrum is referenced to the AUT's plane.

- The field distribution over the AUT's plane is determined by taking the Fourier transform of the previous plane-wave spectrum.

- The field distribution is spatially filtered by setting the samples outside the AUT aperture to zero.

- The filtered field distribution is inverse Fourier transformed back to the spectral domain, obtaining a new plane-wave spectrum where the noise power has been greatly reduced.

After presenting all the points of the proposed method, we analyze the signal-to-noise ratio improvement that can be achieved. The definition of filtering employed in the method appears in Equation (12):

$$
F_{\mathrm{i}}(x, y)=\left\{\begin{array}{ll}
1 & (x, y) \in \omega_{\mathrm{A}} \\
0 & (x, y) \in \omega_{\Gamma}-\omega_{\mathrm{A}}
\end{array},\right.
$$

where $\omega_{T}$ and $\omega_{A}$ represent the reconstructed region and the AUT region depicted in Figure 2. $D_{x}$ and $D_{y}$ are the $x$ and $y$ dimensions of the reconstructed surface, and $A_{x}$ and $A_{y}$ represent the maximum length of the AUT in each direction.

The variation of the maximum signal level in the plane-wave spectrum due to filtering is negligible. As a result, the signal-tonoise ratio improvement remains equal to the noise reduction. The noise power in the plane-wave spectrum without applying any kind of filtering is given by Equation (5). The only unknown quantity needed to specify the improvement is thus the variance of the noise 


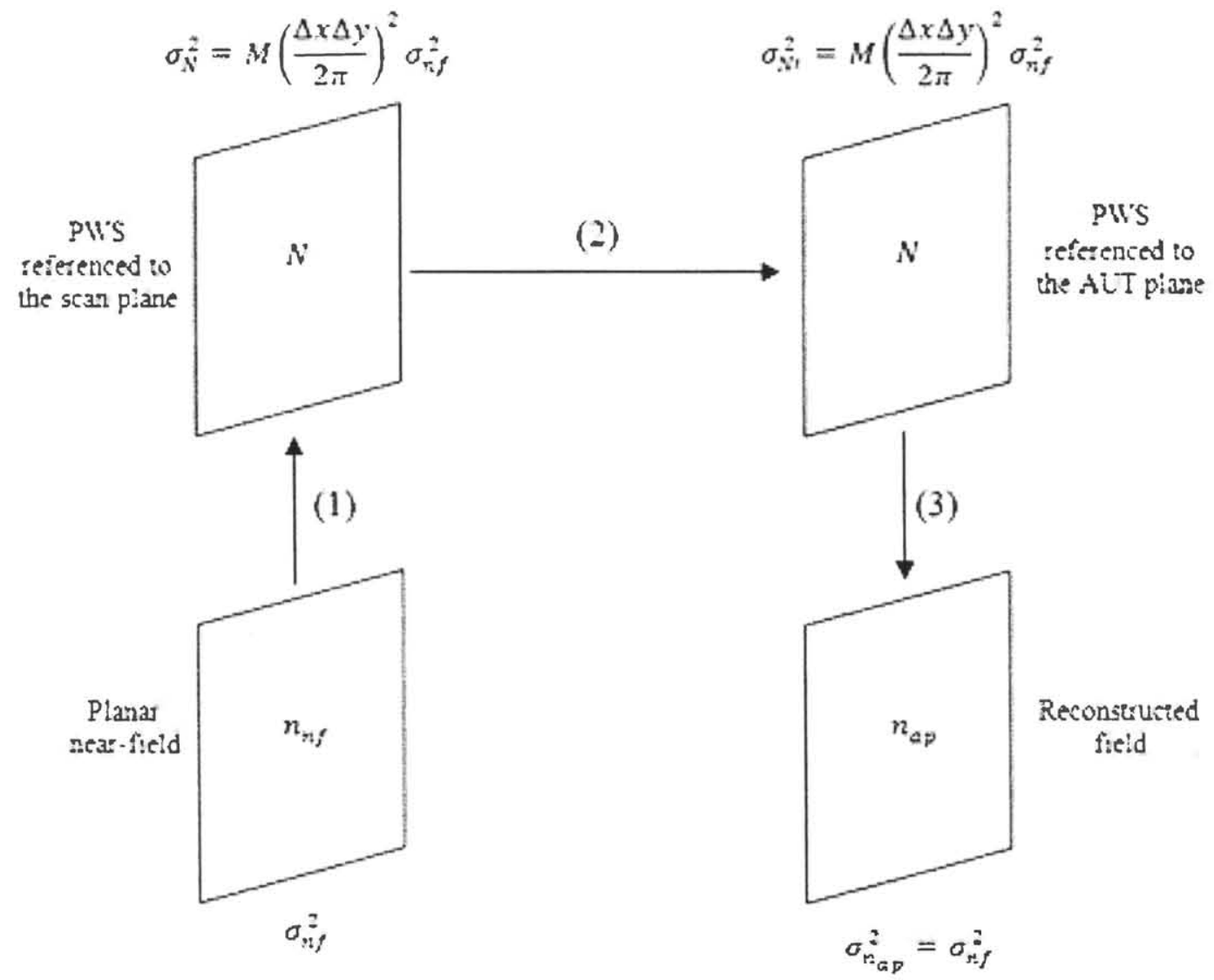

Figure 1. The statistical properties of the noise in the field back-propagation.

in the plane-wave spectrum obtained after spatial filtering. This new plane-wave spectrum is determined as follows:

$$
\begin{array}{r}
N^{\prime}\left(k_{x}, k_{y}, 0\right)=\frac{\Delta x \Delta y}{2 \pi} \sum_{r=1}^{M} F_{1}\left(x_{r}, y_{r}\right) n_{a p}\left(x_{r}, y_{r}, 0\right) e^{j\left(k_{x} x_{r}+k_{y} y_{r}\right)} \\
=\frac{\Delta k_{x} \Delta k_{y}}{2 \pi}\left(\frac{\Delta x \Delta y}{2 \pi}\right)^{2} \sum_{r=1}^{M_{w_{i}}} \sum_{m=1}^{M_{k}} e^{j k_{s, m} d} \sum_{i=1}^{M} n_{n f}\left(x_{i}, y_{i}, d\right) e^{j\left(k_{x, m} x_{i}+k_{y, m} y_{i}\right)} \\
e^{-j\left(k_{x, m} x_{r}+k_{y, m} y_{r}\right)} e^{j\left(k_{x} x_{r}+k_{y} y_{r}\right)}
\end{array}
$$

The noise power of this final quantity, denoted as $\sigma_{N^{\prime}}^{2}$, can be calculated as Equation (14) indicates:

$$
\begin{aligned}
\sigma_{N^{\prime}}^{2} & =R_{N^{\prime}}(0,0)=E\left[N^{\prime}\left(k_{x}, k_{y}, 0\right) N^{\prime *}\left(k_{x}, k_{y}, 0\right)\right] \\
& =M\left(\frac{\Delta x \Delta y}{2 \pi}\right)^{2}\left(\frac{\Delta x M_{x}}{2 \pi}\right)^{2} \Delta k_{x}^{2}\left(\frac{\Delta y M_{y}}{2 \pi}\right)^{2} \Delta k_{y}^{2} \frac{M_{\omega_{A}}}{M} \sigma_{n f}^{2} \\
& =M\left(\frac{\Delta x \Delta y}{2 \pi}\right)^{2} \frac{S_{\omega_{A}}}{D_{x} D_{y}} \sigma_{n f}^{2}
\end{aligned}
$$

where $S_{\omega_{A}}$ is the area of $\omega_{A}$. The signal-to-noise ratio improvement achieved with the proposed spatial filtering method can therefore be calculated using Equations (5) and (14):

$$
\Delta S N R_{S F, t h}=\frac{S N R_{S F}}{S N R_{W F}}=\frac{S / \sigma_{N^{\prime}}^{2}}{S / \sigma_{N}^{2}}=\frac{D_{x} D_{y}}{S_{\omega_{A}}}
$$

In this section, the desired field is assumed to be concentrated on the AUT region. Nevertheless, this assumption is not completely correct. A small field contribution always exists outside the AUT. On the other hand, because the measurement is performed in planar near field, there is a truncation error that expands the field over the AUT plane when the measured data are back-propagated. In cases where filtering is applied, as defined in Equation (12), a portion of the desired signal is cancelled. This cancellation introduces an error, mainly in the sidelobes, in the final plane-wave spectrum. To avoid this negative effect, spatial filtering over a larger area must be employed to account for all of the desired data. However, this new filter integrates more noise power. A compromise is

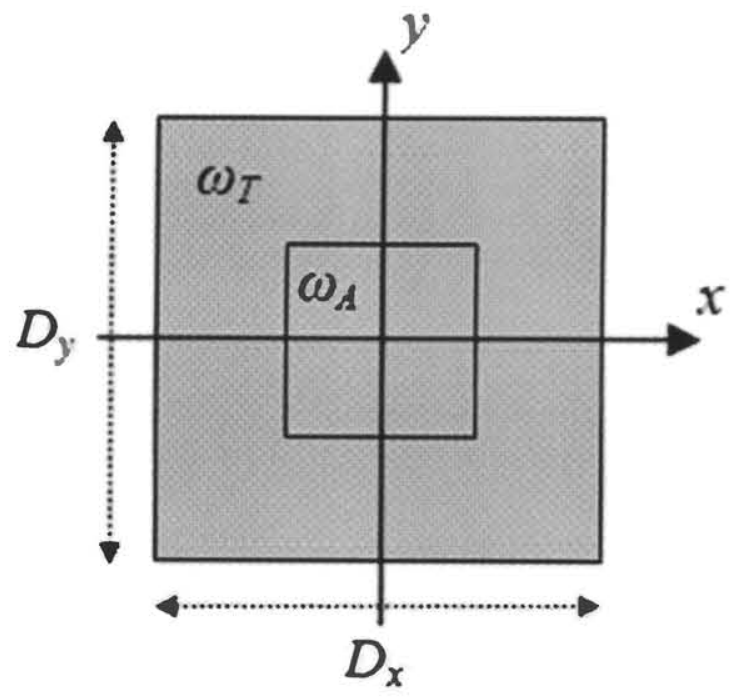

Figure 2. Regions of interest in the reconstructed domain. 


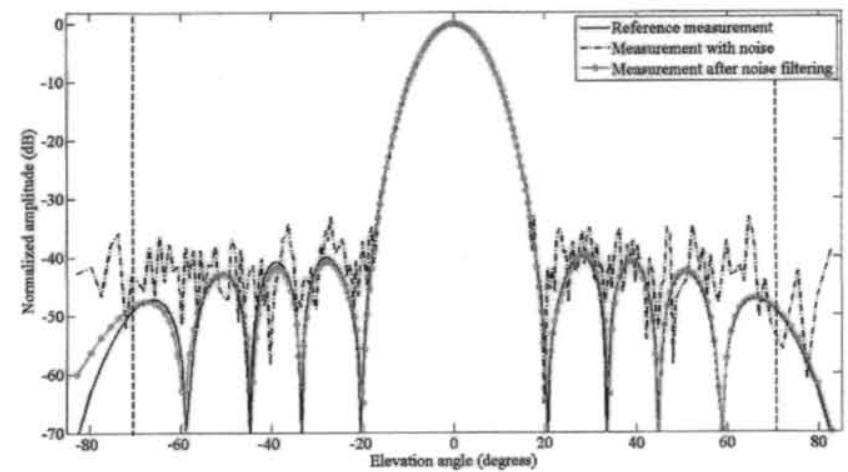

Figure 3. A comparison of the simulated reference antenna pattern and the simulated antenna pattern with and without noise filtering.

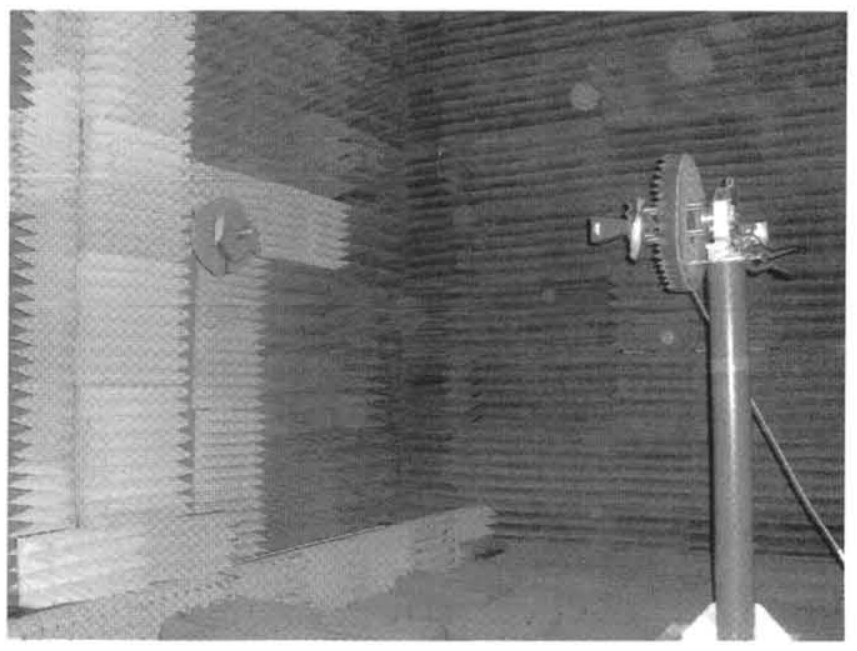

Figure 4. Measurement of a pyramidal horn antenna in the UPM planar near-field range.

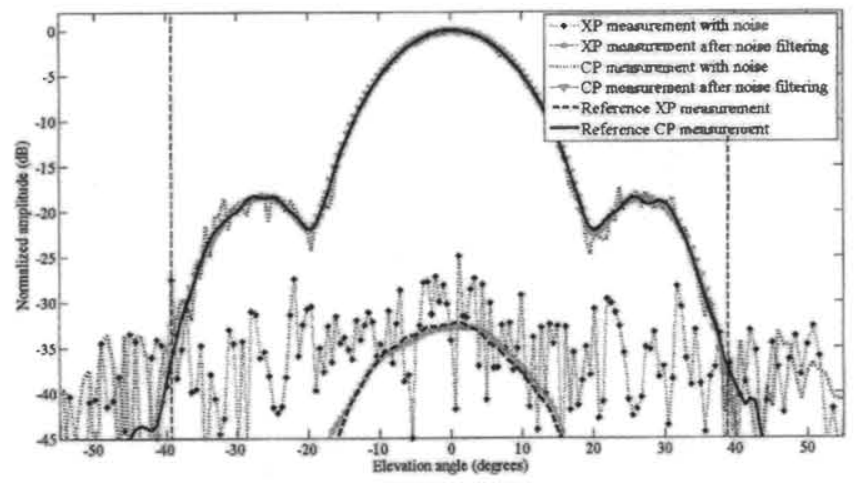

Figure 5. A comparison of the measured reference antenna pattern and the measured antenna pattern with and without noise filtering.

therefore required between the noise reduction and an accurate plane-wave spectrum representation.

\section{Numerical results}

To validate the proposed method, two different examples are presented. The first example takes as input data the values of a simulation of a planar acquisition. The last example uses informa- tion from an actual measurement in the planar near-field range of the Technical University of Madrid (UPM).

\subsection{Simulated Near-Field Data Containing Signal and Noise}

In the first example, a simulation that considered both noise and the contribution of the AUT is presented. The AUT was composed of $14 \times 14$ infinitesimal dipoles with a Hamming excitation. The objective of this form of excitation is to obtain a low-sidelobe far-field pattern, and to better see the effectiveness of the algorithm. The separation between the dipoles was $0.5 \lambda(12 \mathrm{GHz})$, and the planar near-field samples were spaced at $0.5 \lambda$ intervals. These samples were taken over a scan surface of $D_{x}$ by $D_{y}=3.125 \mathrm{~m}$ by $3.125 \mathrm{~m}$, and the distance from the measurement surface to the AUT was $d=20 \lambda$. Once the planar acquisition of the infinitesimal dipole array was simulated, taking into account all previous specifications, Gaussian noise with $50 \mathrm{~dB}$ less power than the maximum of the simulated data was added. Next, the proposed method was applied. Figure 3 shows a cut of the radiation pattern, showing that after filtering a portion of noise in the reconstructed field, it was possible to very effectively reduce the noise in the farfield pattern and to retrieve the reference pattern within the reliable region. The signal-to-noise ratio improvement could be calculated by applying Equation (15), and it was equal to $25.04 \mathrm{~dB}$.

\subsection{Measured Planar Near-Field Data}

In another example, which used the data from an actual measurement, data were obtained by using the planar-range measurement system in the anechoic chamber at the Technical University of Madrid (UPM). For the experiment, the probe and the AUT consisted of a corrugated conical-horn antenna and a $5 \mathrm{~cm}$ by $7 \mathrm{~cm}$ pyramidal-horn antenna. The antennas were separated by $1.57 \mathrm{~m}$. Once both antennas were mounted onto positioners (see Figure 4), a measurement over a $2.4 \mathrm{~m}$ by $2.4 \mathrm{~m}$ acquisition plane, with a spatial sampling equal to $0.43 \lambda(13 \mathrm{GHz})$ was recorded. Gaussian noise with $30 \mathrm{~dB}$ less power than the maximum of the acquired data was added computationally. The noise power was chosen to be large, so as to ensure a negligible measurement noise. The farfield obtained from the measured data without additive noise can thus be used as a reference to compare results before and after noise filtering. As in the preceding example, after obtaining the corrupted data, the method to improve the signal-to-noise ratio was employed. In this case, there was a large truncation error, so a filtering window larger than the AUT's dimensions was required $(0.3 \mathrm{~m} \times 0.3 \mathrm{~m})$. The improvement achieved with this filtering was equal to $18.06 \mathrm{~dB}$. Figure 5 depicts a cut of the radiation pattern where it was possible to see that improvement both in the co-polar and cross-polar components.

\section{Conclusions}

We have presented a simple and efficient method to improve the signal-to-noise ratio in far-field results obtained from planar near-field measurements. Because the method is based on backpropagating the field from the scan plane to the AUT's plane, statistical analysis of the noise in this transformation has also been explored. The results of this last study were used to deduce a theoretical expression for the signal-to-noise ratio improvement. 
Finally, the effectiveness and ease of use for the additive improvement of the method were evaluated through application to both simulated and measured near-field data

\section{Acknowledgement}

This work was supported in part by the FPU Scholarships of the Spanish Education Ministry and the Spanish Projects CROCANTE number TEC2008-06736-C03-01/TEC and TERASENSE number CSD2008-00068.

\section{References}

1. A. D. Yaghjian, "An Overview of Near-Field Antenna Measurements," IEEE Transactions on Antennas and Propagation, AP34, 1, January 1986, pp. 30-44.

2. R. C. Johnson, H. A. Ecker, and J. S. Hollis, "Determination of Far-Field Antenna Patterns From Near-Field Measurements," Proceedings of the IEEE, 61, 12, December 1973, pp. 1668-1694.

3. P. Petre and T. K. Sarkar, "Planar Near-Field to Far-Field Transformation Using an Equivalent Magnetic Current Approach," IEEE Transactions on Antennas and Propagation, AP-40, 11, November 1992, pp. 1348-1356.

4. T. K. Sarkar and A. Taaghol, "Near-Field to Near/Far-Field Transformation for Arbitrary Near-Field Geometry Utilizing an Equivalent Electric Current and MoM," IEEE Transactions on Antennas and Propagation, AP-47, 3, March 1999, pp. 566-573.

5. A. C. Newell, "Error Analysis Techniques for Planar Near-Field Measurements," IEEE Transactions on Antennas and Propagation, AP- 36, 6, June 1988, pp. 754-768

6. L. A. Muth, "Displacement Errors in Antenna Near-Field Measurements and Their Effect on the Far-Field," IEEE Transactions on Antennas and Propagation, AP-36, 5, May 1988, pp. 581-591.

7. H. Hojo and Y. Rahmat-Samii, "Error Analysis for Bi-Polar Near-Field Measurement Technique," IEEE International Symposium on Antennas and Propagation, London, ON, June 24-28, 1991, pp. 1442-1445.

8. A. C. Newell and A. D. Yaghjian, "Study of Errors in Planar Near-Field Measurements," IEEE International Symposium on Antennas and Propagation, June 1975, pp. 470-473.

9. M. H. Francis and R. C. Wittman, "Sources of Uncertainty for Near-Field Measurements," Proceedings of the Second European Conference on Antennas and Propagation (EuCAP 2007), Edinburgh, November 11-16, 2007, pp. 1-3.
10. J. E. Hansen, Spherical Near-Field Antenna Measurements, London, UK, Peter Peregrinus Ltd., 1988.

11. E. B. Joy, "Near-Field Range Qualification Methodology," IEEE Transactions on Antennas and Propagation, AP-36, 6, June 1988 , pp. 836-844.

12. A. C. Newell and C. F. Stubenrauch, "Effect of Random Errors in Planar Near-Field Measurement," IEEE Transactions on Antennas and Propagation, AP-36, 6, June 1988, pp. 769-773.

13. J. B. Hoffman and K. R. Grimm, "Far-Field Uncertainty Due to Random Near-Field Measurement Error," IEEE Transactions on Antennas and Propagation, AP-36, 6, June 1988, pp. 774-780.

14. J. Romeu, L. Jofre, and A. Cardama, "Far-Field Errors Due to Random Noise in Cylindrical Near-Field Measurements," IEEE Transactions on Antennas and Propagation, AP-40, 1, January 1992, pp. 79-84.

15. Romeu and L. Jofre, "Effect of Random Errors in Cylindrical Near-Field Measurements," IEEE International Symposium on Antennas and Propagation, London, ON, June 24-28, 1991, pp. $1450-1453$

16. J. Romeu, L. Jofre, and A. Cardama, "An Approximate Expression to Estimate Signal-to-Noise Ratio Improvement in Cylindrical Near-Field Measurements," IEEE Transactions on Antennas and Propagation, AP-42, 7, July 1994, pp. 1007-1010.

17. P. Koivisto, "Reduction of Errors in Antenna Radiation Patterns Using Optimally Truncated Spherical Wave Expansion," Progress in Electromagnetic Research, PIER-47, 2004, pp. 313333.

18. L. J. Foged and M. Faliero, "Random Noise in Spherical NearField Systems," Proceedings 2009 Antenna Measurement. Techniques Association, AMTA, Salt Lake City, UT, November 1-6, 2009, pp. 135-138.

19. F. Jensen and A. Frandsen, "On the Number of Modes in Spherical Wave Expansion," Proceedings 2004 Antenna Measurement. Techniques Association, AMTA, Stone Mountain Park, GA, October 2004, pp. 489-494.

20. J. J. H. Wang, "An Examination of the Theory and Practices of Planar Near-Field Measurement," IEEE Transactions on Antennas and Propagation, AP-36, 6, June 1988, pp. 746-753.

21. E. Martini, O. Breinbjerg, and S. Maci, "Reduction of Truncation Errors in Planar Near-Field Aperture Antenna Measurements Using the Gerchberg-Papoulis Algorithm," IEEE Transactions on Antennas and Propagation, AP-56, 11, November 2008, pp. 34853493. Ais) 